\title{
Tests of Stepping as Indicators of Mobility, Balance, and Fall Risk in Balance-Impaired Older Adults
}

\author{
Be-long Cho, MD, ${ }^{* \ddagger}$ Diane Scarpace, $R N, N P,{ }^{*}$ and Neil B. Alexander, $M D^{* \dagger}$
}

OBJECTIVES: To determine the relationships between two tests of stepping ability (the maximal step length (MSL) and rapid step test (RST)) and standard tests of standing balance, gait, mobility, and functional impairment in a group of at-risk older adults.

DESIGN: Cross-sectional study.

SETTING: University-based laboratory.

PARTICIPANTS: One hundred sixty-seven mildly balance-impaired older adults recruited for a balance-training and fall-reduction program (mean age 78, range 65-90).

MEASUREMENTS: Measures of stepping maximally (MSL, the ability to maximally step out and return to the initial position) and rapidly (RST, the time taken to step out and return in multiple directions as fast as possible); standard measures of balance, gait, and mobility including timed tandem stance (TS), tandem walk (TW, both timing and errors), timed unipedal stance (US), timed up and go (TUG), performance oriented mobility assessment (POMA), and 6-minute walk (SMW); measures of leg strength (peak knee and ankle torque and power at slow and fast speeds); self-report measures of frequent falls ( $>2$ per 12 months), disability (Established Population for Epidemiologic Studies of the Elderly (EPESE) physical function), and confidence to avoid falls (Activity-specific Balance Confidence (ABC) Scale). Spearman and Pearson correlation, intraclass correlation coefficient, logistic regression, and linear regression were used for data analysis. RESULTS: MSL consistently predicted a number of selfreport and performance measures at least as well as other standard balance measures. MSL correlations with EPESE physical function, ABC, TUG, and POMA scores; SMW;

From the * Mobility Research Center, Division of Geriatric Medicine, Department of Internal Medicine, University of Michigan, Ann Arbor, Michigan; ${ }^{\dagger}$ Ann Arbor Veterans Affairs Health Care System Geriatric Research Education and Clinical Center, Ann Arbor, Michigan; and ${ }^{\ddagger}$ Department of Family Medicine, College of Medicine, Seoul National University, Seoul, Korea.

Funding received from National Institute on Aging (NIA) Claude Pepper Older Adults Independence Center Grant AG08808, NIA Grant AG10542, and Department of Veterans Affairs Research and Development.

Dr. Alexander is a recipient of the K24 Mid-Career Investigator Award in Patient-Oriented Research AG109675 from NIA.

Address correspondence to Neil B. Alexander, MD, Geriatrics Center, 1500 East Medical Center Drive, Ann Arbor, MI 48109.

E-mail: nalexand@umich.edu and peak maximum knee and ankle torque and power were at least as high as those correlations seen with TS, TW, or US. MSL score was associated with the risk of being a frequent faller. In addition, the six MSL directions were highly correlated (up to 0.96), and any one of the leg directions yielded similar relationships with functional measures and a history of falls. Relationships between RST and these measures were relatively modest.

CONCLUSION: MSL is as good a predictor of mobility performance, frequent falls, self-reported function, and balance confidence as standard stance tests such as US. MSL simplified to one direction may be a useful clinical indicator of mobility, balance, and fall risk in older adults. J Am Geriatr Soc 52:1168-1173, 2004.

Key words: postural control; aged; falls; fall risk; balance; mobility; gait

$\mathbf{R}$ elationships between falls, balance, and gait are sufficiently complex that a number of balance and gait tests have been developed to predict fall risk. The majority of these tests, including measures such as unipedal stance (US), ${ }^{1}$ tandem stance (TS), ${ }^{2}$ functional reach, ${ }^{3}$ and dynamic posturography, ${ }^{4}$ assess the ability to maintain stance in response to a postural challenge. This ability to maintain stance is based on a series of corrective arm, trunk, and leg responses while maintaining feet in place. Other performance test batteries have been developed to assess overall mobility, the presence of balance and gait disorders, and, ultimately, fall risk, including the timed up and go test (TUG), ${ }^{5}$ the performance oriented mobility assessment (POMA), ${ }^{6}$ and the Berg balance test. ${ }^{7}$ However, none of these tests focus on the ability to take a step, a response often used when strategies to maintain stance are inadequate. Trips and slips are the most common contributors to older adult falls, ${ }^{8}$ and responding to a slip or trip requires taking a step.

Recent studies have examined stepping in older adults. In response to externally applied perturbations of stance, older adults differ from young adults in the number of steps used to respond ${ }^{9}$ and in the ability to successfully recover with a step in response to increasing perturbation 
magnitudes. ${ }^{10}$ Age-related slowing in volitional stepping (in response to a visual or auditory stimulus) is particularly common when the older adult has to change leg or direction. ${ }^{11-13}$ Older adults with a history of falls use stepping responses more frequently than nonfallers, particularly at lower levels of postural disturbances. ${ }^{14}$ Fallers also exhibit slowed voluntary stepping, particularly when having to change leg or direction. ${ }^{12,15}$

Worse scores on two measures of stepping (maximal step length (MSL) and rapid step test (RST)) have been reported in older adults than younger adults and in balanceimpaired than unimpaired older adults. ${ }^{13}$ MSL is the maximum distance that one can step out and still return to stance, whereas RST is the time needed to take 24 steps of near-MSL length in various directions. The current study reports on the baseline values of MSL and RST in a large cohort of at-risk older adults enrolled in a prospective controlled study to determine the effect of exercise on improving stepping ability and ultimately reducing falls. To validate MSL and RST as measures of balance and fall risk, MSL and RST performance were correlated with other measures of fall risk, gait and mobility, and disability (TUG, POMA, 6-minute walk (SMW), Established Population for Epidemiologic Studies of the Elderly (EPESE) physical function, Activity-Specific balance Confidence (ABC) Scale, and leg strength). These relationships were also examined in other standard balance screening tests (US, TS, tandem walk (TW)), given that MSL and RST may eventually be used as screening tests for dynamic balance. The relationships between leg strength and MSL and RST were also examined, given that leg strength is likely a critical factor underlying MSL and RST performance. The hypothesis was that the MSL and RST, as measures of maximal and rapid stepping, are as good as typical stance measures of balance such as US and TS in predicting performance on standard measures of gait, mobility, and leg strength and self-report measures of frequent falls, disability, and confidence in avoiding falls.

\section{METHODS}

\section{Subjects}

One hundred sixty-seven community-dwelling subjects aged 65 and older were recruited with a particular focus on independent residents of congregate housing facilities. Advertisements and presentations at each facility were used to recruit participants for a 1-year prospective trial of exercise to reduce falls. A nurse practitioner screened subjects, who were excluded if they were medically unstable (e.g., having ongoing chest pain or marked dyspnea) or were unable to participate in standing exercise due to severe spinal or lower extremity joint pain. Because the goal was to include participants who might best benefit from a standing balance exercise program, participants who were too unstable (unable to stand for 30 seconds with feet together and eyes open without human or device assistance) or, conversely, too able (could tandem walk 10 feet without errors and stand on one leg for more than 30 seconds with eyes open) were excluded. Subjects with substantial cognitive impairment (Mini-Mental State Examination score $<24$ out of 30 ) were also excluded. Of 258 potential participants screened, 49 (19\%) were excluded for reasons given above, and $39(15 \%)$ declined because of the exercise program time commitment.

\section{Measurements}

All timing was performed using a hand-held stopwatch.

\section{Measures of Maximal and Rapid Stepping}

\section{Maximal Step Length}

While keeping their arms across their chests, subjects stepped out maximally with one leg, maintaining the stance leg in the initial position, and then returned to their initial stance position in one step. Adhesive-backed ruler tape was affixed to the floor to the front, back, and each side of the subject to assist in measurement. After three practice trials of submaximal stepping in each leg-direction (right, left/ front, side, back), MSL was defined as the average step length over a series of five trials in each leg-direction (five trials for each of six leg-directions, making a total of 30 trials).

\section{Rapid Step Test}

Subjects were instructed to step out with one leg as quickly as possible and return the leg to the initial starting position. The experimenter gave the command for each leg and direction (e.g., "left-front") in a random order that included four steps in each of six leg-directions, for a total of 24 repetitions. Subjects first practiced random sequences of six steps on each leg and then proceeded to the 24-step protocol. Subjects were required to step a distance of at least $80 \%$ of their MSL for all leg-directions; this distance was marked with a taped line on the floor. An error was defined as failure of the foot to touch or exceed the taped line; loss of balance, usually indicated by marked trunk motion such that the experimenter approached the subject to assist in preventing a fall; failure to return to initial position; multiple steps; noncompliance with leg-direction; or failure to keep arms crossed. Total time to complete 24 repetitions and total errors occurring during the 24repetition trial were recorded.

\section{Measures of Balance, Gait, and Mobility $T S, U S$, and TW}

Three trials of each test were performed, for up to 60 seconds for TS and 30 seconds for US. For TS, subjects stood heel to toe; for US, subjects stood with their lifted foot approximately 2 inches from the medial malleolus of the stance foot without making foot-to-malleolus contact. For US, subjects stood first on their preferred leg, then the opposite leg, and then their preferred leg again, while keeping their arms across their chest. For TW, subjects were required to walk heel to toe along a 10 -foot line. They were asked to walk as quickly as they could without errors. Errors included not walking heel to toe, stepping off the gait line, and losing balance. The performance time (and number of errors for TW) of three trials for performance of each task was averaged for analyses. Any subject unable to perform TS, US, or TW was assigned a score of 0 and still included in the analyses. Because lower TW (shorter performance time) represents better performance, inclusion 
of 0 scores for TW necessitated use of the reciprocal of TW in the analyses.

\section{TUG, POMA, and SMW}

Additional standard measures of balance and gait were also measured: TUG (the time taken to rise from a chair, walk 3 meters, turn, and return to a sitting position); $;^{5}$ POMA (a rating of performance of a set of functional balance tasks and gait); ${ }^{6}$ and SMW (the maximum distance walked over 6 minutes).

\section{Measures of Leg Strength \\ Peak Knee and Ankle Torque and Power}

Using an isokinetic dynamometer (Biodex Multisystem 2AP, Biodex Medical, Shirley, NJ) with standard seated positioning and strap restraints, peak isokinetic torque (in $\mathrm{Nm})$ and power (in watts) were tested at slow $(120 \%$ s) and fast $(300 \%)$ speeds for knee extension and flexion and at slow $(60 \%)$ and fast $(120 \%$ s) speeds for ankle dorsiflexion and plantar flexion. Peak was recorded from five repetitions for each joint movement direction in the dominant leg (the leg that the subject felt was strongest or the leg used for kicking a ball). Both torque and power were standardized by height times weight.

Self-Report Measures: Frequent Falls, Disability, and Confidence to Avoid Falls

Subjects were asked about falls in the previous year (those with more than two in the previous year were considered frequent fallers), activities of daily living, higher-order mobility task (Nagi, Rosow-Breslau) disability (EPESE physical function battery ${ }^{16}$ ), and confidence to avoid falls $\left(\mathrm{ABC}\right.$ scale $\left.{ }^{17}\right)$. Falls were defined as unintentionally coming to rest on the ground, floor, or other lower level.

Table 1. Performance on Maximal Step Length (MSL), Rapid Step Test (RST), Balance, and Strength Tests $(\mathbf{n}=$ 167)

\begin{tabular}{lcc}
\hline \multicolumn{1}{c}{ Test } & $\begin{array}{c}\text { Mean } \pm \text { Standard } \\
\text { Deviation }\end{array}$ & $\begin{array}{c}\text { Unable, } \\
\mathrm{n}(\%)\end{array}$ \\
\hline Tandem stance, seconds & $18.5 \pm 22.1$ & $60(35.9)$ \\
Unipedal stance, seconds & $5.6 \pm 7.0$ & $60(35.9)$ \\
Tandem walk & & $68(40.7)$ \\
$\quad$ Time, seconds & $3.7 \pm 6.7$ & \\
Error, n & & $3(1.8)$ \\
RST & $60.1 \pm 18.4$ & \\
Time, seconds & $7.6 \pm 5.8$ & \\
Error, n & $23.3 \pm 6.6$ & $1(0.5)$ \\
MSL, inches & & \\
Strength, Nm torque & $12.0 \pm 6.1$ & \\
$\quad$ Ankle dorsiflexion $(60 \% \mathrm{sec})$ & $8.2 \pm 3.5$ & \\
Ankle dorsiflexion $(120 \% \mathrm{sec})$ & $19.2 \pm 12.7$ & \\
Ankle plantarflexion $(60 \% \mathrm{sec})$ & $15.0 \pm 10.0$ & \\
Ankle plantarflexion $(120 \% \mathrm{sec})$ & $36.7 \pm 20.4$ & \\
Knee extension $(120 \% / \mathrm{sec})$ & $23.4 \pm 13.7$ & \\
Knee extension $(300 \% \mathrm{sec})$ & $21.1 \pm 11.9$ & \\
Knee flexion $(120 \% \mathrm{sec})$ & $21.4 \pm 12.1$ & \\
Knee flexion $(300 \% \mathrm{sec})$ & & \\
\hline
\end{tabular}
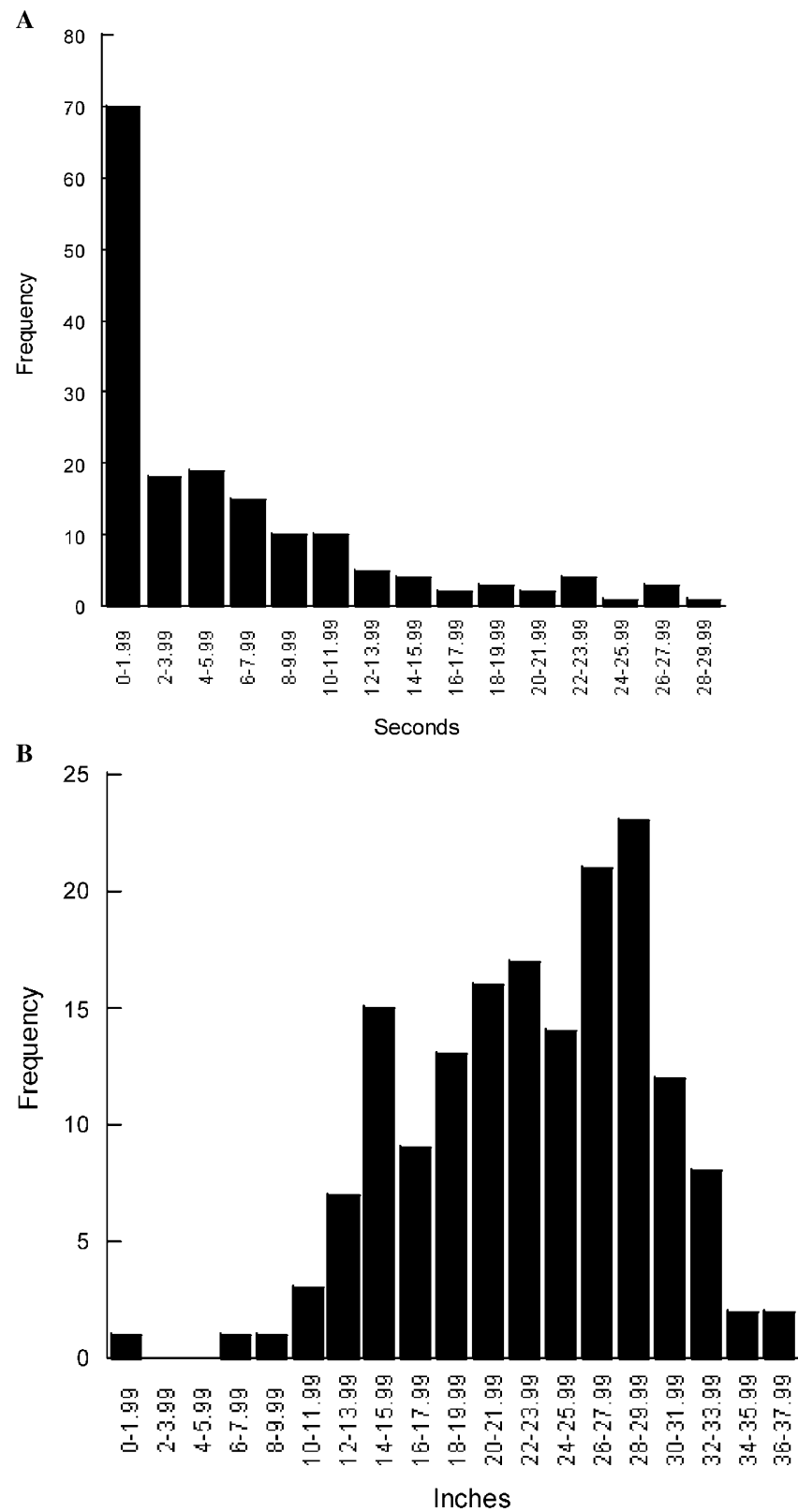

Figure 1. Frequency distribution of A. unipedal stance and B. maximum step length.

\section{Data Analysis}

Because of concern for skewed distributions in US, TS, and TW, Spearman correlation coefficients were used to assess relationships between MSL, RST, and all performance and self-report measurements. Relationships between the six leg-directions of MSL were assessed using repeated measure analysis of variance and Pearson correlation coefficients. Logistic regression was used to analyze whether MSL, RST, or other measures predicted frequent falling (as above, $>2$ falls/12 months), adjusted for age and sex. Because of the differences in units between the different measures, these odds ratios (ORs) for frequent falling are reported according to change in 1 standard deviation. Intraclass correlations (ICCs) were used to evaluate the test-retest reliability of average and single leg-direction MSL. Cronbach alpha was calculated to evaluate the concordance of 
the six leg-directions of MSL. The one-sample Kolmogorov-Smirnov test was used to analyze the normality of MSL and US distributions. All analyses were performed using SPSS version 10.0 (SPSS Inc., Chicago, IL) with the significance level for all analyses set at $P<.05$.

\section{RESULTS}

Participants were mostly Caucasian $(90 \%)$ and female $(77 \%)$, with a mean age \pm standard deviation of $78 \pm 7$ (range 65-90). Twenty-six (16\%) participants admitted to having two or more falls in the previous 12 months and were thus identified as frequent fallers. Mean EPESE physical function score was $4 \pm 3$, but basic activity of daily living limitation was minimal, except for the $19(11 \%)$ who admitted to using a cane or walker occasionally outdoors or for long distances. Mean ABC scale score was $78 \pm 20$, consistent with individuals with mild reduction in balance confidence and with chronic health conditions. ${ }^{18}$ There was a tendency toward mild impairments in functional mobility, balance, and gait. Mean scores were $25 \pm 5$ for POMA, $15 \pm 8$ seconds for TUG, and $1,109 \pm 367$ feet for SMW, further attesting to the tendency for mild impairment.

Table 1 shows the performance data for MSL, RST, and other balance-related tasks. As an indicator of group impairment, a substantial percentage was unable to perform TS $(36 \%)$, US (36\%), or TW ( $41 \%)$, and the mean US was low (less than 6 seconds), but essentially all subjects performed MSL and RST. All of the balance-related tasks were significantly skewed to the bottom of the distribution (by Kolmogorov-Smirnov test, $P<.05$ ), except MSL, which approximated a normal distribution in this sample (Figure 1 for comparison of US and MSL distributions).

Table 2 shows the Spearman correlation coefficients between MSL; RST; other balance, gait, and mobility measures; and the self-report measures. MSL correlated highly with TUG, SMW, POMA, EPESE, and ABC (range $0.58-0.75, P<.01$ ). These correlations were at least as high as those seen with the other balance measures, US, TS, and TW, with values ranging from 0.38 to $0.74(P<.01)$. RST correlations were the poorest, ranging from 0.15 to 0.38 (most $P<.01$ ). MSL also correlated strongly with peak isokinetic ankle dorsi- and plantarflexion and knee extensor and flexor strength at fast and slow speeds (range 0.46$0.55 ; P<.01$; full data available from authors). Again, these correlations were at least as high as those with US, TS, and TW (range 0.27-0.44; most $P<.01$ ), and the strength correlations with RST were lower (range 0.23-0.42; most $P<.01$ ). Correlations were similar for normalized (by height and weight) torque and for power at these joints and speeds. The odds of being a frequent faller (vs the rest of the cohort), after adjusting for age and sex, were significant for MSL as well as for TUG, SMW, TW error, US, TS, and POMA $(P<.05)$ (Table 3$)$. MSL is correlated with height or leg length $(r=0.35-0.37, P<.001)$, but repeating all of the above analyses with normalized MSL does not appreciably alter these correlation outcomes.

The six individual leg-directions for the MSL were highly correlated $(r=0.88-0.96, P<.001)$, and the Cronbach alpha of these leg-directions was high (0.98). Correlations between single leg-direction MSL and key balance (US, TS) and functional measures (EPESE, ABC, TUG, SMW, and POMA) ranged from 0.54 to 0.73 for front movement (either leg), 0.50 to 0.72 for lateral movement (either leg), and 0.54 to 0.75 for back movement (either leg). Any of the leg-directions yielded as high an OR for frequent fallers as mean MSL $(\mathrm{OR}=0.90-0.92$, $P<.01)$. Test-retest reliability of the six leg-directions (and mean MSL) was evaluated in 62 subjects, and the range of ICC was high (for the six leg-directions $=0.79$ 0.85 , for mean MSL $=0.86$, both $P<.001)$. Test-retest reliability of the RST is much lower $(\mathrm{ICC}=0.42, P<.001)$.

\section{DISCUSSION}

MSL consistently predicted a number of self-report and performance measures at least as well as other standard balance measures. MSL correlations with EPESE physical function score, ABC, TUG, POMA, and SMW scores and peak maximum knee and ankle torque and power were at least as high as correlations with TS, TW, or US. MSL was strongly associated with the risk of being a frequent faller. In addition, the six MSL directions were highly correlated

Table 2. Correlations between Maximal Step Length (MSL), Rapid Step Test (RST), Other Balance and Mobility Measures, and the Self-Report Measures

\begin{tabular}{|c|c|c|c|c|c|c|c|c|c|c|}
\hline Measure & MSL & RST & TS & US & TW & TUG & SMW & POMA & EPESE & $A B C$ \\
\hline MSL & 1.000 & & & & & & & & & \\
\hline RST & -.384 & 1.000 & & & & & & & & \\
\hline TS & .617 & -.369 & 1.000 & & & & & & & \\
\hline US & .696 & -.351 & .776 & 1.000 & & & & & & \\
\hline TW & -.629 & .332 & -.758 & -.642 & 1.000 & & & & & \\
\hline TUG & -.679 & .346 & -.485 & -.558 & .564 & 1.000 & & & & \\
\hline SMW & .732 & -.287 & .519 & .527 & -.524 & -.752 & 1.000 & & & \\
\hline POMA & .748 & -.376 & .687 & .738 & -.615 & -.651 & .617 & 1.000 & & \\
\hline EPESE & -.579 & .150 & -.434 & -.531 & .380 & .495 & -.563 & -.545 & 1.000 & \\
\hline ABC & .661 & -.321 & .592 & .586 & -.520 & -.606 & .631 & .637 & -.644 & 1.000 \\
\hline
\end{tabular}

Note: All correlations significant at $P<.01$.

$\mathrm{TS}=$ tandem stance time; US = unipedal stance time; TW = tandem walk time; TUG = timed up and go; SMW = 6-minute walk; POMA = performance-oriented mobility assessment; EPESE = Established Populations for the Epidemiologic Study of the Elderly physical function scale; ABC $=$ activities-specific balance confidence scale. 
Table 3. Odds Ratios for Frequent Falling (Adjusted for Age and Sex)

\begin{tabular}{lcc}
\hline \multicolumn{1}{c}{ Measure } & $\begin{array}{c}\text { Odds Ratio } \\
\text { (95\% Confidence Interval) }\end{array}$ & $P$-value \\
\hline Timed up and go, seconds & $1.70(1.18-2.45)$ & .005 \\
6-minute walk, feet & $0.53(0.34-0.83)$ & .005 \\
Tandem walk error, $\mathrm{n}$ & $2.93(1.39-6.18)$ \\
Maximal step length, inches & $0.52(0.32-0.84)$ \\
Unipedal stance, seconds & $0.38(0.17-0.84)$ \\
Tandem stance, seconds & $0.50(0.27-0.92)$ \\
Performance oriented mobility assessment, $\mathrm{n}$ & $0.65(0.42-0.99)$ \\
Tandem walk, seconds & $1.47(0.94-2.29)$ \\
Established Population for the & $1.42(0.93-2.16)$ \\
$\quad$ Epidemiologic Study of the Elderly, $\mathrm{n}$ & & .008 \\
Activities-specific balance confidence test, $\mathrm{n}$ & $0.71(0.46-1.11)$ \\
Rapid step test error, $\mathrm{n}$ & $1.21(0.79-1.85)$ \\
Rapid step test, seconds & $0.98(0.63-1.53)$ \\
\hline
\end{tabular}

(up to 0.96), and any one of the leg-directions yielded similar relationships with functional measures and risk of frequent falling. This latter result may mean that testing of all six directions may be unnecessary. Relationships with these measures and reliability involving RST were relatively modest.

Given the relationships found in these data, what exactly do MSL and RST measure? MSL might be a measure of how much strength (and power) the subject can consistently generate (average output), not just in stepping out, but also in the ability to safely make the return step. There were no clear differences in the relationships between MSL and strength (or power) developed rapidly or more slowly at the knee or ankle. Correlations between maximal rapid strength and MSL might be increased by performing MSL in one direction (without a return step) and by analyzing MSL as the longest (instead of the average) of five trials. ${ }^{19}$ Also, MSL most likely involves perceived disability and confidence in determining a safe step length that the subject can execute while maintaining an acceptable risk of loss of balance. Previous fallers accordingly limit their step length. Whether the step out or the return step is the most critical component of MSL is the subject of an ongoing biomechanical study.

In contrast, RST most likely involves these factors as well, but also may involve a greater cognitive component. In the RST, subjects must choose the leg-direction, similar to a choice-reaction time task. They must also be able to hear properly the leg-direction instruction, see the target line, limit their errors in execution, and return to their original starting position as quickly as possible. To some extent, the RST also depends upon how quickly the experimenter gives the next leg-direction command. In the present study, a sizable number of errors (mean RST error of 8) occurred, perhaps suggesting difficulty (and possibly variability) with task performance, although error rate on RST correlated poorly (0.12) with performance time. Previous reports of higher correlations between RST and clinical measures $(0.51-0.8)$, and of higher RST test-retest reliability (0.87-0.9) may be the result of inclusion of young and balance-unimpaired older adults who have more consistent, error-free performance. ${ }^{13}$
The study sample selected, based on screening for bipedal stance, UP stance, and TW performance represents a relevant and commonly found target group for a number of reasons. First, fewer than $10 \%$ of community-dwelling older adults cannot stand for 30 seconds with feet together. ${ }^{20}$ Second, $50 \%$ of a sample of at-risk older adults enrolled in exercise programs to reduce falls were initially unable to stand on one leg, ${ }^{2}$ slightly higher than the $36 \%$ rate reported here. Finally, difficulty in performing TW is present in at least half of community-dwelling older adults and is associated with increased risk of hip fracture. ${ }^{21}$ The findings that essentially all participants in the present study were able to perform the MSL and the relatively normal distribution of the MSL scores support the potential application of MSL testing in communitydwelling older adults with various levels of balance impairment.

Based on the present study, MSL appears to be valid and reliable in an older adult group at risk for falls. The MSL appears to be at least as good as a common measure of standing balance and predictor of falls (US), ${ }^{1}$ and with a more normalized distribution than US, MSL can provide a more quantitative score for a wider range of at-risk older adults. Other recent data confirm a decline in MSL over the life span, the high correlations between MSL and other functional measures, and that a single leg-direction can predict overall performance. ${ }^{19}$ Use of a one-leg-direction MSL as a clinical test holds promise. Although the entire MSL and RST battery takes less than 10 minutes to administer, the time requirement for a single leg-direction MSL is minimal. Whether MSL can prospectively identify fallers and whether MSL is sensitive to exercise-induced improvements in balance are key questions to be answered in an ongoing intervention.

\section{ACKNOWLEDGMENTS}

The authors also thank Jodi Medell for her work on developing the MSL and RST, and Wendy Champoux, Ravi Goswami, Julie Grunawalt, Mark Hofmeyer, Tisha Moore, Theo Morrow, Martina Nabozny, Linda Nyquist, Rebecca Olson, Diane Scarpace, Debra Strasburg, and David Wilson 
for their assistance in data collection. The authors also thank Dr. Phil Park and Professor Sungil Cho for their statistical advice and Jeffrey Halter, MD, of the Michigan Geriatrics Center for his sponsorship of visiting overseas faculty.

\section{REFERENCES}

1. Vellas BJ, Wayne SJ, Romero L et al. One-leg balance is an important predictor of injurious falls in older persons. J Am Geriatr Soc 1997;45:735-738.

2. Rossiter-Fornoff JE, Wolf SL, Wolfson LI et al. A cross-sectional validation study of the FICSIT common data base static balance measures. J Gerontol A Biol Sci Med Sci 1995;50A:M291-M297.

3. Duncan PW, Studenski S, Chandler J et al. Functional reach: predictive validity in a sample of male veterans. J Gerontol 1992;47:M93-M98.

4. Wolfson L, Whipple R, Derby CA et al. Gender differences in the balance of healthy elderly as demonstrated by dynamic posturography. J Gerontol 1994;49:M160-M167.

5. Podsiadlo D, Richardson S. The timed 'Up \& Go': A test of basic functional mobility for frail elderly persons. J Am Geriatr Soc 1991;39:142-148.

6. Tinetti ME, Speechley M, Ginter SF. Risk factors for falls among elderly persons living in the community. N Engl J Med 1988;319:1701-1707.

7. Berg KO, Wood-Dauphinee SL et al. Measuring balance in the elderly: Validation of an instrument. Can J Public Health 1992;83:S7-S11.

8. Berg W, Alessio H, Mills E et al. Circumstances and consequences of falls in independent community-dwelling older adults. Age Ageing 1997;26:261-268.

9. Luchies CW, Alexander NB, Schultz AB et al. Stepping responses of young and old adults to postural disturbances: Kinematics. J Am Geriatr Soc 1994; 42:506-512.
10. Thelen DG, Wojcik LA, Schultz AB et al. Age differences in using a rapid step to regain balance during a forward fall. J Gerontol A Biol Sci Med Sci 1997;52A:M8-M13.

11. Luchies CW, Schiffman J, Richards LG et al. Effects of age, step direction, and reaction condition on the ability to step quickly. J Gerontol A Biol Sci Med Sci 2002;57A:M246-M249.

12. Lord SR, Fitzpatrick RC. Choice stepping reaction time: A composite measure of falls risk in older people. J Gerontol A Biol Sci Med Sci 2001;56A:M627M632.

13. Medell J, Alexander N. A clinical measure of maximal and rapid stepping in older women. J Gerontol A Biol Sci Med Sci 2000;55A:M429-M433.

14. Chandler JM, Duncan PW, Studenski SA. Balance performance of the postural stress test. Comparison of young adults, healthy elderly, and fallers. Phys Ther 1990;70:410-415.

15. Dite W, Temple VA. A clinical test of stepping and change of direction to identify multiple falling adults. Arch Phys Med Rehabil 2002;83:1566-1571.

16. Smith LA, Branch LG, Scherr PA et al. Short-term variability of measures of physical function in older people. J Am Geriatr Soc 1990;38:993-998.

17. Powell LE, Myers AM. The Activities-specific Balance Confidence scale. J Gerontol A Biol Sci Med Sci 1995;50A:M28-M34.

18. Myers AM, Fletcher PC, Myers AH et al. Discriminative and evaluative properties of the Activities-specific Balance Confidence (ABC) Scale. J Gerontol A Biol Sci Med Sci 1998;53A:M287-M294.

19. Lindemann U, Bauerle C, Muche R et al. Age-related differences in balance, strength, and motor function. Eur J Ger 2003;5:15-22.

20. Guralnik JM, Simonsick EM, Ferrucci L et al. A short physical performance battery assessing lower extremity function: Association with self-reported disability and prediction of mortality and nursing home admission. J Gerontol 1994;49:M85-M94.

21. Dargent-Molina P, Favier F, Grandjean H et al. Fall-related factors and risk of hip fracture: The EPIDOS prospective study. Lancet 1996;348:145-149. 